\title{
The NOAO Publications Tracking Program: Developing Policies \& Procedures for Usage, Acknowledgment, and Citation of Data
}

\author{
Sharon Hunt ${ }^{1, \star}$ and Dara J. Norman ${ }^{1}$ \\ ${ }^{1}$ National Optical Astronomy Observatory, 950 N. Cherry Ave., Tucson, AZ 85719, USA
}

\begin{abstract}
The National Optical Astronomy Observatory (NOAO) has a complex publications tracking program, which is coordinated by the NOAO Librarian. We track publications that use data from multiple observatories (Kitt Peak National Observatory [KPNO] in Tucson, Arizona, and Cerro Tololo Inter-American Observatory [CTIO] in La Serena, Chile, as well as non-NOAO telescopes to which we grant community access at other observatories); multiple telescopes and instruments; multiple observing programs, including survey programs; and multiple datasets in the Science Data Archive (SDA) at NOAO. The growing SDA and new user services, such as the Data Lab, ensure that the use of NOAO archival datasets will become even more widespread and important for scientific discovery and research. Accurate and complete publication tracking is essential for reporting to our funding agencies, developing our programs, and determining our scientific contributions and impact. Setting policies to guide authors in specifying and acknowledging usage of NOAO data is an essential element in our ability to determine and document relevant publications. Developing systems to track the use of our telescope data and archival datasets is another critical element. New policies to track the use of our telescope data are being developed, new systems to track the use and reuse of datasets within our particular institutional context are being explored, and methods of linking our publications data with our dataset usage data are being devised. Updated policies for the proper and ethical usage, acknowledgement, and citation of our archival data are being written and reviewed.
\end{abstract}

\section{Introduction}

A high-quality telescope bibliography is an essential tool for developing and reporting on observatory programs; at the same time, acknowledgments of data usage are essential for making explicit the scientific contributions of an observatory, particularly to funding agencies. The National Optical Astronomy Observatory (NOAO) has sought to develop our publications tracking policies and procedures to improve the efficiency and accuracy of our publications tracking, to provide guidance to telescope data users on appropriate acknowledgments in publications, and to develop and adhere to standards of professional ethics relating to data usage.

\footnotetext{
^e-mail: hunt@noao.edu ORCID: 0000-0002-5858-2002
} 


\section{Identifying and Documenting Publications That Utilize Data from NOAO Facilities and Services}

NOAO has a multifaceted publications tracking program, as we track the usage of telescope data from multiple sites, telescopes and instruments, survey programs, and data products and services. We maintain telescopes at two sites (Kitt Peak National Observatory in Tucson, Arizona, USA, and Cerro Tololo Inter-American Observatory in La Serena/Cerro Tololo, Chile). We also grant communityaccess telescope time on several non-NOAO telescopes. We collect astronomical data from multiple telescope/instrument combinations and survey programs and store and make accessible data in the Science Data Archive at NOAO.

Our literature search and analysis process is complex, utilizing multiple keywords and searching strategies due to the wide scope of our program and the variant designations (e.g., KPNO vs. Kitt Peak, CTIO vs. Cerro Tololo; Mayall vs. Kitt Peak 4m, etc.) used by authors for our resources. We also contend with the issue of incomplete documentation on the data used in some publications. Because of these factors, we face the need to analyze closely many publications to weed out false positives and determine the specifics of data usage. The documentation of the many parameters relating to use of our data (telescope/instrument combination; survey program; dataset; investigators) is timeconsuming and labor-intensive.

Our revised policies and procedures address these challenges in identifying and documenting relevant publications in two ways: providing explicit and readily accessible acknowledgements statements and utilizing the NOAO Proposal ID as a source of details of data usage.

\subsection{Acknowledgments Guidelines}

Standardized and comprehensive acknowledgment of the use of NOAO data by authors is vital to properly tracking publications. In addition, ethical standards would suggest that there should be recognition of other people's work through proper acknowledgment of data usage. Our first goal was to develop explicit guidelines and statements on how authors can stipulate and acknowledge use and reuse of our telescope data, data products, and archival datasets. We then made these policies readily accessible through our NOAO Library Publications Acknowledgements web page. This resource serves as a centralized repository for NOAO publications policies and procedures, with links from all appropriate web pages (e.g., Science Data Archive at NOAO; proposals for telescope usage web pages; and publications listings) so that authors can easily find acknowledgments information and so that acknowledgments statements are standardized in all publications. This web page contains acknowledgements statements specific to particular telescopes, programs, and data products and services and thus provides explicit guidance to authors on our expectations relating to the use of NOAO data and their responsibilities as users of this data.

\subsection{NOAO Proposal Identifiers}

The NOAO Proposal Identifier (NOAO Prop. ID) is a unique number given to each proposal requesting time on our telescopes. See Figure 1 for an example of a NOAO Prop. ID web page and the information it provides.

This unique identifier offers a mechanism to streamline and enhance the literature searching and analysis process. We now request the usage of this identifier in the acknowledgments section of publications. It provides us with the needed details on investigators, dates, telescopes, datasets, and instruments and negates the necessity of individually capturing specifics of data usage from a particular publication. It often supplies more information on the data usage than may be explicitly mentioned in a publication, and it lessens the amount of time and effort required to track and analyze publications. 


\section{Proposal Information for 2014A-oo73}

PI: Mukremin Kilic, University of Oklahoma, kilic@ou.edu

Address: Physics Department, 440 W Brooks Street, Norman, OK 73019, USA

CoI: Sara Barber, University of Oklahoma

CoI: Buell Januzzi, NOAO

CoI: Arjun Dey, NOAO

CoI: Peter Stetson, National Research Council of Canada

Title: A Search for Habitable Planets Around White Dwarfs

Abstract: A large fraction of white dwarfs (WDs) may host planets in their habitable zones. Here we propose to take advantage of the unique capability of DECam on the CTIO $4 \mathrm{~m}$ to perform a pre-LSST transit survey that is capable of detecting the first planet in the habitable zone of a WD. We propose to obtain DECam time-series photometry of the CFHT Legacy Survey (CFHTLS) Wide2 field to search for planetary, substellar, and stellar companions to more than 500 WDs. Thanks to the 3 square degree field of view of DECam, for the first time in history, a large number of WDs can be observed at the same time, which is essential for discovering transits. We will use the original data taken 8 years ago for the CFHTLS and our stacked DECam images to identify the WDs based on their colors and proper motions. Given the observing window from the ground, if every WD has an Earth-like planet in its habitable zone, we expect to find up to 3 planets in this initial survey. In addition to planets, we will also constrain the frequency of eclipsing substellar and stellar companions to WDs, which has important implications for the LSST. Our stacked dataset will also provide a significantly improved WD luminosity function for the Galactic disk and halo.

Figure 1. NOAO proposal web page, providing information on observing program, investigators, and telescope/instrument combinations.

The NOAO Prop. ID ties the data used in a publication to specific investigators and will be attached to any re-use of this data in another publication. It thus serves to make the contribution of the investigator explicit and easily trackable.

We are developing new systems to track the use and reuse of datasets within our particular institutional context. The NOAO Prop. ID is the mechanism through which we can link our proposals database, datasets in the Science Data Archive at NOAO, and our publications database, allowing us to combine and analyze in a seamless manner the details of NOAO data usage (Figures 2 and 3).

\begin{tabular}{|c|c|c|c|c|c|c|c|c|c|c|c|c|c|c|c|}
\hline $\begin{array}{c}\text { Proposal } \\
\text { ID }\end{array}$ & $\frac{\text { Survey }}{\text { ID }}$ & $\begin{array}{l}\text { Release } \\
\text { date }\end{array}$ & $\begin{array}{l}\text { Observing } \\
\text { date }\end{array}$ & UT - & PI & $\underline{\text { RA }}$ & Dec & Telescop & Instrument & Filter & Exposure & $\frac{\text { Observation }}{\text { type }}$ & $\begin{array}{l}\text { Observing } \\
\text { mode }\end{array}$ & Processing & Product \\
\hline$\frac{2014 \mathrm{~A}-}{\underline{0073}}$ & ? & $\begin{array}{l}2015- \\
08-03\end{array}$ & $\begin{array}{c}2014-02- \\
02\end{array}$ & $\begin{array}{c}2014-02-03 \\
05: 03: 21.505\end{array}$ & Kilic & 135.758700 & -4.583333 & $\mathrm{ct} 4 \mathrm{~m}$ & decam & $\begin{array}{c}\mathrm{g} \\
\text { DECam } \\
\text { SDSS } \\
\text { c0001 } \\
4720.0 \\
1520.0\end{array}$ & 30.0 & object & imaging & RAW & image \\
\hline
\end{tabular}

Figure 2. Science Data Archive at NOAO web page with information on observing program

\section{NOAO Data Policies}

Usage of NOAO archival datasets is becoming even more widespread and important for scientific discovery and follow-up, making proper acknowledgment complex. Policies on standards of professional

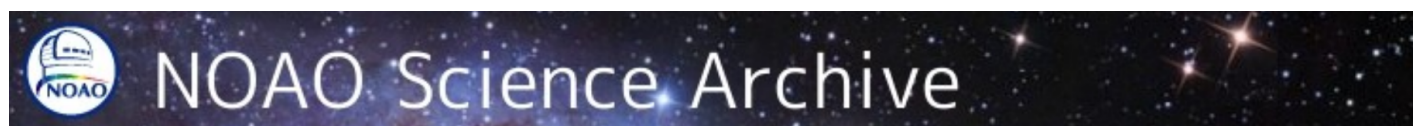

Figure 3. Citation from NOAO online telescope bibliography using data from observing program of NOAO Prop. ID 2014A-0073. The NOAO Prop. ID was listed in the acknowledgments section of this publication. The citation, NOAO proposal web page, and Science Data Archive at NOAO are linked through the NOAO Prop. ID. 
Belardi, C., ... Dey, A., et al. 2016, MNRAS, 462, 2506, The DECam minute cadence survey - I 2016MNRAS.462.2506B

Blanco +DECam; NOAO Prop. ID 2014A-0073, PI: M. Kilic; Science Data Archive at NOAO

Figure 4. Citation from NOAO online telescope bibliography using data from observing program of NOAO Prop. ID 2014A-0073. The NOAO Prop. ID was listed in the acknowledgments section of this publication. The citation, NOAO proposal web page, and Science Data Archive at NOAO are linked through the NOAO Prop. ID.

ethics and conduct with respect to data usage are not always known and adhered to. Furthermore, revised policies are needed to reflect our expansion into open-access digital science platforms, services, and software (e.g., Data Lab, ANTARES).

We seek to ensure that NOAO data policies are consistent with (or ahead of) other observatories and data archives/centers and that they reflect proper ethical standards for data use and citation. We regularly review policies of other observatories and data archives/centers and revise and add statements for new data products, services, and software as needed. We propose modifications and updates to suggested responsible use of archival data, possibly including acknowledgment of original PI(s), for review by relevant managers. We propose to work with publication groups to modify instructions to journal referees to look for and point out failures to properly acknowledge data use, software, and services.

\section{Conclusions}

Since we have developed our NOAO Library Publications Acknowledgments web page, we have seen steadily increasing usage of our suggested acknowledgments statements in publications. Providing a readily accessible reference source detailing our expectations regarding acknowledgments has aided authors in specifying the usage of our data. This in turn has streamlined our publications tracking process. Our documentation of NOAO data usage is more efficient and more complete with the increased usage of NOAO Prop. IDs in publication acknowledgments sections, as the Prop. ID provides us with a wealth of information on the observing program that gathered the data used in a specific publication. The use of our standardized acknowledgments statements more readily identifies NOAO's and specific investigators' contributions.

\section{Acknowledgments}

The National Optical Astronomy Observatory (NOAO) is operated by the Association of Universities for Research in Astronomy (AURA) under cooperative agreement with the National Science Foundation (NSF). 